\title{
Portable impedance measurement system based on Embedded
}

\author{
System \\ LUO Xiaohui ${ }^{1, a}$ \\ ${ }^{1}$ Chongqing College of Electronic Engineering Chongqing 401331 \\ a.luohui54@yahoo.com.cn
}

\begin{abstract}
AD5934 and MSP430F169 based on the design of a portable impedance test system, the system uses MSP430F169 as the main control chip, through the $\mathrm{I}^{2} \mathrm{C}$ control module to complete impedance impedance measurement, and through the buttons to control the operation process and test results through LCD display, impedance test chip AD5934 is highly integrated, low cost. The accurate measurement of impedance can be achieved; the main control chip MSP430F169 with low power consumption, high efficiency advantages, rich on-chip resources. The system has the advantages of small size, low power consumption and high efficiency, and can be used for measuring the electric conductivity of the common capacitor. The measurement of LC series parallel circuit shows that the system can accurately measure the impedance values at different frequencies.
\end{abstract}

Key words: impedance; measurement; portable; embedded

\section{Introduction}

Impedance measurement has been widely used in many fields, such as agriculture, chemical industry, medicine and so on. [1]. With the deepening of scientific research, the accuracy and efficiency of the measurement of resistance is also increased by [2]. Impedance measurement method is diverse, the resonance method using RLC series parallel circuit in the resonance of the physical behavior to measure impedance, more close to the actual conditions of the components, the system is used to measure the impedance of the resonance [3].Traditional resistance measurement system is large, and the same operation, the system uses the AD5934 as the impedance test chip, MSP430F169 based control processing chip to achieve a portable and low power consumption requirements.

\section{Hardware Design}

The hardware system includes the following diagram, power module (ADP3303), MSP430F 169 controller module, impedance measurement module, AD5934 LCD module (LCD12864), a key control module, communication module (MAX232)



Fig. 1 system hardware structure diagram

\section{1 impedance measurement module}

The impedance measurement module uses AD5934. The digital frequency waveform generator is 
integrated in the chip. The $12 \mathrm{bit}$, 250KSPS is used to measure the response of the digital to analog converter (ADC): at the same time, the ADC sampling data can be carried out at the 1024 point discrete time DFT transform DSP. DDS frequency synthesizer based on the need to generate a sinusoidal signal to be measured impedance, the external impedance can be resistive, inductive, capacitive, etc.. The response signal of external impedance by on-chip A/D converter ADC sampling, in order to ensure that the system is linear and avoid signal response signals over the ADC range, at the time of measurement should be an external feedback resistor, feedback resistor selection range is calculated according to the measured impedance is obtained, and then the DFT transform of DAC data by DSP. DFT algorithm returns a real part (R) and a virtual part (I) at each frequency.

Due to the ADC5934 in the output sine signal will produce DC component, the colleagues of the four amplitude sinusoidal voltage corresponding to the four output impedance. Will affect the accuracy of small impedance measurement, and therefore need to be in the external signal by eliminating the influence of DC component and output impedance on the measurement [4]. The effects of low noise, track input and output, CMOS operational amplifier AD8606[5] optimized signal path to eliminate the output impedance of AD5934 on impedance measurement.

\section{2 embedded control module}

MSP430F169 the peripheral circuit is used to form the whole system control module. MSP430 Series MCU is a 16 bit ultra low power MCU produced by TI company, which integrates rich on-chip resources, on-chip watchdog, analog comparator, timer, LCD driver and ADC at the same time, the supply voltage is low and flexible operation clock design so that it can achieve low power consumption. MSP430F169 MCU integrates FLASH ROM $2 \mathrm{~KB}$ and RAM 64KB. The power supply voltage is $1.8-3.6 \mathrm{~V}, 16$ bit RISC structure, $125 \mathrm{~ns}$ instruction cycle, 86 bit parallel ports, and so on. It has the characteristics of low power consumption, low power consumption and high efficiency.

\section{3 system software design}

According to the working principle of AD5934 microcontroller program, complete the analysis of frequency scanning and impedance, then the measurement results will be sent via the $\mathrm{I}^{2} \mathrm{C}$ bus to the MSP430F169 microcontroller, microcontroller to send back the results of the measurement were analyzed after treatment in the liquid crystal display, or first bit machine.

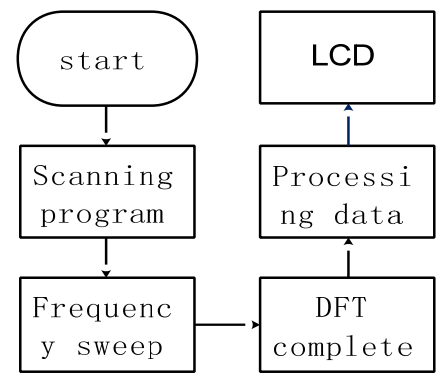

Fig. 2 program flow chart

\section{4 experimental results and analysis}

Using this system to test the LC series parallel circuit, the circuit model can also simulate the biological impedance [6]. As shown in Figure 3 circuit of the equivalent model, $\mathrm{L}$ and $\mathrm{C}$ are connected in series with the OUT and IN pins, the resistance R is generated by the $\mathrm{L}, \mathrm{C}$ itself, the test $\mathrm{A}, \mathrm{B}$, and the impedance of the two ends of the circuit. 


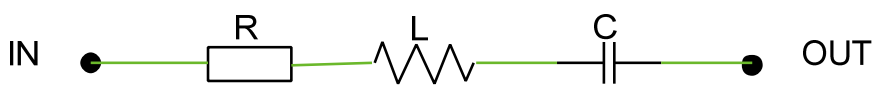

Fig. 3 RLC series circuit

Then $Z=R+j\left(\omega L-\frac{1}{\omega C}\right),|Z|=\sqrt{R^{2}+\left(\omega L-\frac{1}{\omega C}\right)^{2}}$. When $\omega L=\frac{1}{\omega C}$, then $\omega=\sqrt{L C}$ the minimum value of impedance, at this time $f=\frac{\omega}{2 \pi}=\frac{1}{2 \pi \sqrt{L C}}$. When the impedance is the hour, the corresponding frequency is the resonant frequency, that is, when the circuit frequency reaches the resonant frequency of the circuit, the circuit impedance value is the smallest. Experiment time circuit, series, $100 \mathrm{uH}$ inductor, $110 \mathrm{uF}$ capacitor, At this point $f=\frac{\omega}{2 \pi}=\frac{1}{2 \pi \sqrt{L C}}$, when the frequency is $50328 \mathrm{HZ}$, the impedance value is minimum, and the measurement results are shown in Table 1.

Table 1 test results

\begin{tabular}{|l|l|l|l|}
\hline Excitation frequency/kHz & Impedance value $/ \Omega$ & Excitation frequency $/ \mathrm{kHz}$ & Impedance value $/ \Omega$ \\
\hline 10 & 123.56 & 55 & 90.16 \\
\hline 15 & 118.24 & 60 & 90.32 \\
\hline 20 & 113.35 & 65 & 96.5 \\
\hline 25 & 109.07 & 70 & 104.12 \\
\hline 30 & 105.32 & 75 & 111.47 \\
\hline 35 & 101.71 & 80 & 119.22 \\
\hline 40 & 98.18 & 85 & 126.13 \\
\hline 45 & 94.05 & 90 & 133.21 \\
\hline
\end{tabular}

The corresponding curves of frequency and impedance are shown in Figure 4.

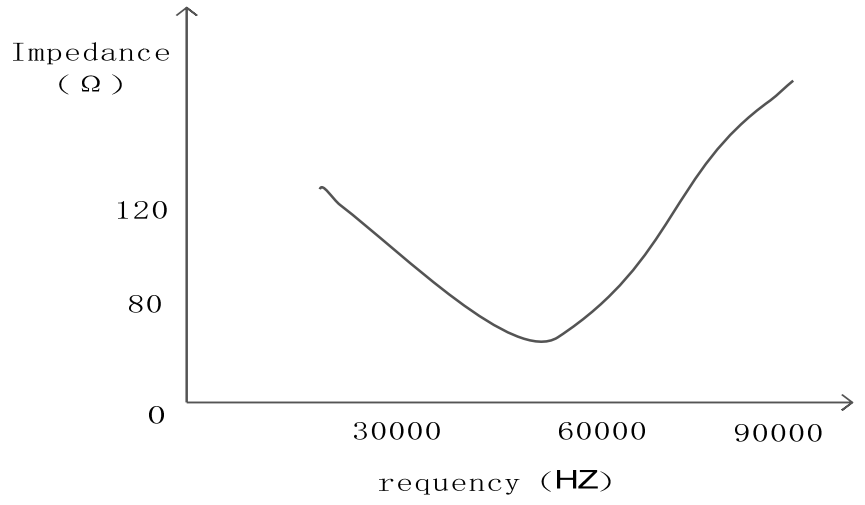

Fig. 4 characteristics of impedance and frequency variation

When the map and measurement data can be properly excitation signal is 50350, corresponding to the lowest point of the impedance, that is, the resonant frequency of the frequency error of $21 \mathrm{HZ}$, the relative error of $0.04 \%$.

\section{5 conclusions}

In this paper, a low power consumption, high efficiency, on-chip resource rich MSP430F169 
chip with low cost, highly integrated impedance test chip AD5934 combined with a portable impedance test system. The impedance value of the measured object can be measured, and the resonance frequency, the response frequency and the impedance variation can be measured. The relative error of resonance frequency measurement. The measurement system can be applied to the unknown impedance of the ordinary, but also by biological impedance measurement of bio impedance model, widely used, the whole system has the advantages of small volume, complete functions, low cost design, intelligent, accurate measurement.

\section{Reference}

[1] product information - impedance analyzer impedance measurement and role of [J]. radio and television technology, 2001 (2); 137-138.

[2] J.Ferreira,F.Seoane,A.Ansede,etal.AD5933-Based Spectrometer for Electical Bioimpedance Applications[J].Journal of physis:conference series,2010,224:1742-6569.

[3] Jerzy Hoja,Grzegorz Lentka .Interface circuit for impedance sensors using two specialized single-chip Microsystems[J].journal of sensors and Actuators A:physical,2010,163:191-197.

[4] Lin Yan ,Liu Wen Ding. Low-power Design of Impendance Measurement System Based on MSO430F169[A].IEEE International Conference on CASE[C].2011,1:252-254.

[5] Wen Xinhua, Yan Fei, anton. Development of portable impedance instrument based on[J].AD5933, intelligent instrument and sensor technology,2013,21 (4): 1090-1092.

[6] Zhang Ningning. Characteristic analysis and application of RLC series/parallel resonant circuit[J],value engineering.2012,31 (14):36-37. 